\title{
RIDGE AUGMENTATION USING AUTO-LOGOUS CONCENTRATED GROWTH FACTORS (CGF) ENRICHED BONE GRAFT MATRIX (STICKY BONE) VERSUS GUIDED BONEO REGENERATION USING NATIVE COLLAGEN MEMBRANE IN HORIZONTALLY DEFICIENT MAXILLA. (RANDOMIZED CLINICAL TRIAL)
}

\author{
Sarah AbdAllah Aboelela*, Hesham Fattouh ${ }^{* *}$ and Mohammed Atef Abdel Rasoul ${ }^{* * *}$
}

\begin{abstract}
Aim: The aim of the study is to introduce an easy and predictable method for horizontal bone augmentation in maxillary bone ridge using Autologous Concentrated Growth Factors (CGF) enriched bone graft matrix (sticky bone) in contrary with guided Bone regeneration using native collagen membrane.
\end{abstract}

Methodology: A total of 28 patients presenting with Cawood Class IV ridge classification (2-4mm residual alveolar width) were included in the study. 14 patients received horizontal augmentation using sticky bone prepared from a 1:1 mixture of Auto logous and anorganic bovine bone minerals covered by a concentrated growth factors (CGF) membrane. The other 14 patients received lateral augmentation using guided bone rejuvenation by a mixture of 1:1 Auto logous and anorganic bovine bone minerals covered by a native collagen membrane (sausage technique). CBCT scans were taken immediately postoperative and after 6 months to measure the horizontal bone gain in both groups.

Results: For native collagen group, the median lateral gain measured at 2, 5 and $10 \mathrm{~mm}$ from the alveolar crest was 2.55 with range $(1.43,4.34) \mathrm{mm}, 2.61$ with range $(0.02,4.71) \mathrm{mm}$ and 1.15 with range $(-2.88,4.89) \mathrm{mm}$, respectively. While for CGF group, the median lateral bone gain measured at 2,5 and $10 \mathrm{~mm}$ from the alveolar crest was 1.54 with range $(-0,97,2.93) \mathrm{mm}, 2.8$ with range $(-2.01,5.27) \mathrm{mm}, 2.97$ with range $(-1.32,5.4) \mathrm{mm}$, respectively. Mean horizontal bone width was statistically significant within each group, and comparing both groups the difference was also statistically significant.

Conclusion: Within the limitations of the study, horizontal ridge augmentation using sticky bone covered by CGF membrane is a simple technique with unpredictable results compared to guided bone regeneration using native collagen membrane.

* Master of Oral Implantologyn, Cairo University

** Assistant Professor, Faculty of Dentistry, Cairo University

*** Assistant Professor of Oral and Maxillofacial Surgery, Cairo University 


\section{INTRODUCTION}

Tooth extraction is one of the common dental procedures. Generally, the healing of the bony socket done by different manners. However, even with uneventful healing, the bony defect that results as a consequence of tooth loss will only become partially restored. Synchronous with bone healing in the socket, there is also well documented, resorption of the bony ridges. Juodzbalys et al 2013 Structural and functional alterations of the soft and hard tissues of the maxillary ridge occur over time after tooth loss and can directly influence the therapeutic alternatives. Alveolar ridge resorption in partially edentulous patients can intervene with correct insertion of dental implant. Buser et al (2011) Inadequate quantity and quality of residual alveolar ridge is a challenge in achieving implant primary stability which is important for successful osseointegration also presence of healthy adequate bone volume at the potential implant recipient site, is essential in order to obtain long term success of implant restoration. Hämmerle et al 2008., Manuel et al 2009 Lindhe et al 2006 suggested that the greatest amount of bone loss is in the horizontal dimension and occurs mainly on the buccal aspect of the ridge. There is also loss of vertical ridge height. Studies have shown that most of the resorption occurs during the first 3 months of healing and two thirds of the hard and soft tissue changes were occurred, although dimensional changes can be observed up to 1 year $r$ more after tooth loss. Such changes result in nearly $50 \%$ reduction of the bucco-palatal dimension of the alveolar ridge Schropp et al 2003. Depending on the type of atrophy, different treatment modalities have been attempted for horizontal alveolar bone defect Augmentation, including use of particulates and block grafting materials, guided bone regeneration (GBR), distraction osteogenesis, growth and differentiation factors.

\section{AIM OF STUDY}

The aim of the study is to introduce voluble and predictable method for horizontal bone augmentation in maxillary bone ridge using autologous Concentrated Growth Factors (CGF) nriched bone graft matrix (sticky bone) in contrary with guided bony regeneration using collagen membrane.

\section{PATIENTS AND METHOD}

This study was carried on twenty-eight patients suffering from maxillary partial or total edentulism with insufficient bone width to permit implant placement. Patients were selected from the clinic of Oral and Maxillofacial, Surgery Department, Faculty of Oral and Dental medicine, Cairo University. Each patient was interviewed to obtain a medical and dental history. The protocol for this parallel designed trial was reviewed and approved by the ECs [Ethical Committees], Faculty of Dentistry, University, in terms of scientific content and adherence to relevant research and human subjects' regulations. The ethical committee additionally examined and approved particular informed consent forms (Arabic and English versions), participant learning, enrollment materials, any requested documentation, and any future adjustments. The treatment procedures, aim of the study, possible side effects, and treatment alternatives were thoroughly explained to all the participants.

\section{Inclusion criteria}

Candidates with total or partial edentulous maxillary ridge with horizontal bone defect ranging from 2-4 mm (Cawood class III, IV) and a minimum of $8 \mathrm{~mm}$ bony vertical dimension measured from the alveolar crest to the floor of the nasal cavity or to the sinus floor were enrolled in the present study.

\section{Exclusion criteria:}

- A significant disease or pathology involving the bones.

- Smoker's patients. 
- Patients who have a systemic condition that may impede their ability to heal normally.

- Psychiatric issues

- Implant problems are linked to a history of radiation therapy for head and neck neoplasia or implant site bone augmentation.

- Pathology of immunodeficiency, bruxism

\section{Preoperative Assessment}

A preoperative assessment for all enrolled candidate was carried out including history taking, examination clinically and radiographically. A Cone Beam Computed Tomography (CBCT) scan was ordered as a final investigation. On tomographic slices perpendicular to the longitudinal axis of the alveolar crest, bone width measurements were taken., $2 \mathrm{~mm}, 5 \mathrm{~mm}$ and $10 \mathrm{~mm}$ from the top of the crest, At the planned implant sites. Figure1.
The patients were randomly divided into 2 equal groups using block randomization with stratification (block :4) using a formula on Microsoft Excel Software. The first group Enriched Bone Graft Matrix (Sticky Bone) to horizontal defect of maxilla and second group GBR with mechanically stabilized resorbable membrane (sausage technique). Both received augmentation using 1:1 mixture of 50:50 Auto logous and ABBM bone graft. The same surgeon did all of the operations.

\section{GBR SAUSAGE GROUP:}

A resorbable membrane * was customized by a pattern that adjusted to the graft's volume, and considering that the margin of the membrane labially to be fixed between the roots of the neighboring teeth. The membrane was firmly settled to at least at two points on the palatal and labial sides with titanium bone tacks*. Figure2

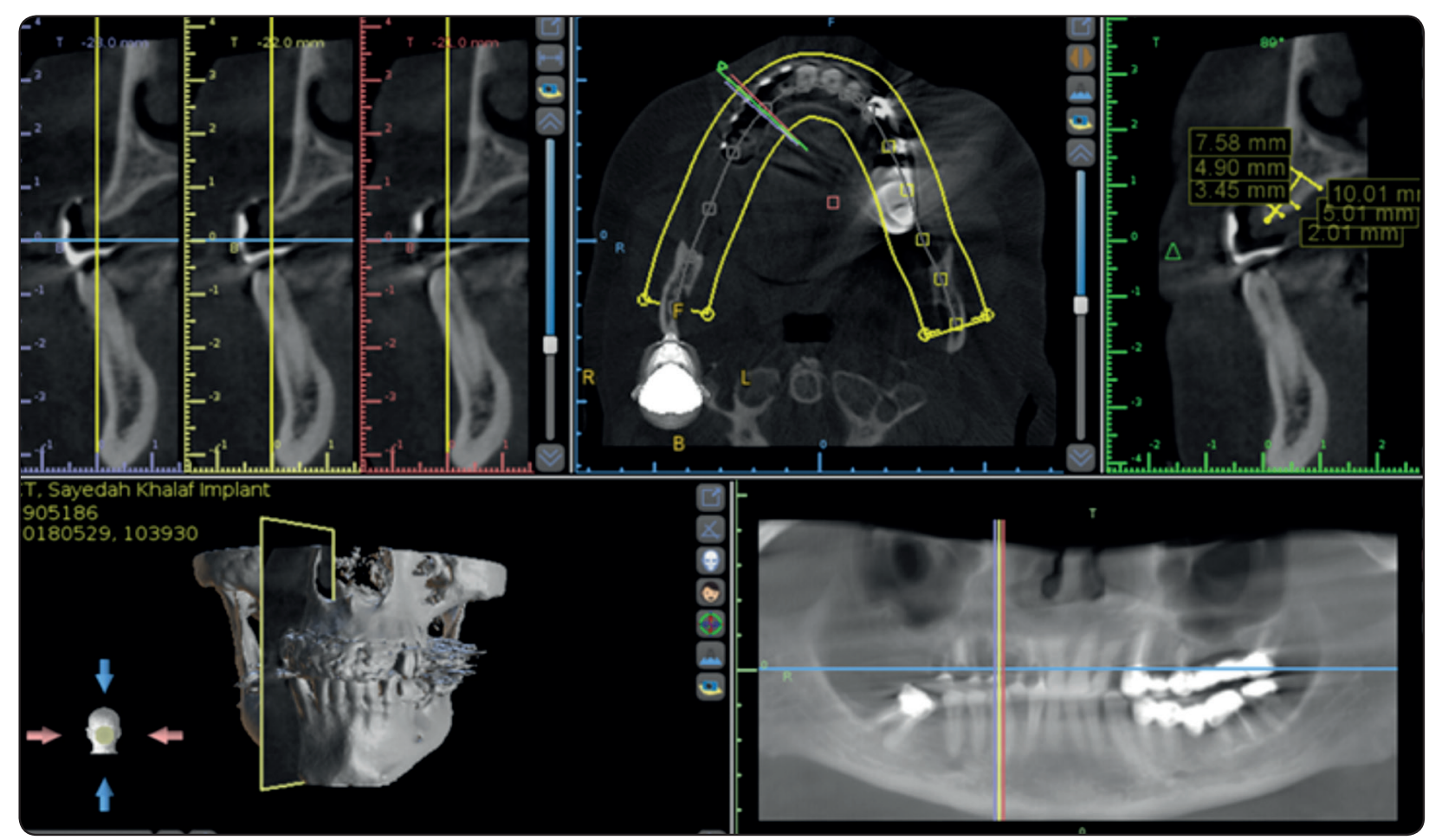

Fig. (1) preoperative measurement taking from CBCT

\footnotetext{
* Bio-Gide®Resorbable Bilayer Membrane, Geistlich Pharma AG, Wolhusen, Switzerland

** Tacs by Botiss biomaterials/GMBH
} 


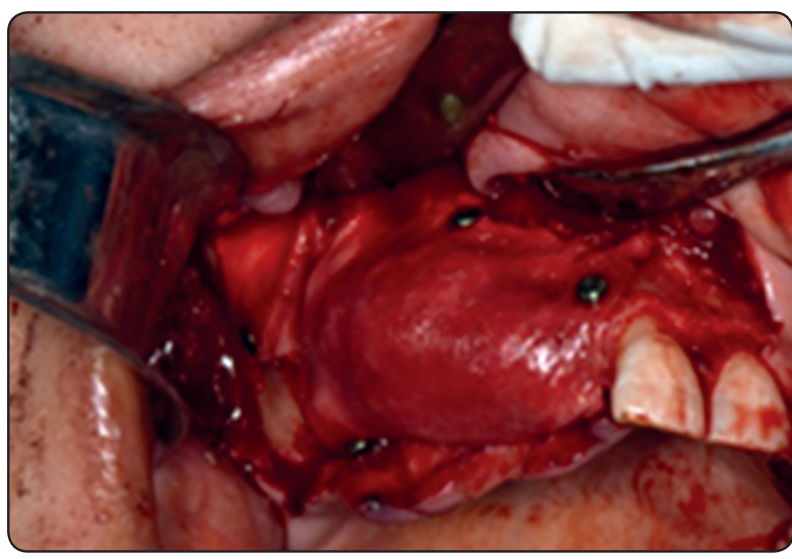

Fig. (2) membrane was fixed by tacks sausage group

\section{CGF GROUP:}

\section{The Preparation Of "Sticky Bone" and Cgf Membrane}

\section{Sticky Bone Preparation}

$10 \mathrm{CC}$ of venous blood was collected from a vein in the patient's forearm and inserted in non-coated plastic vacutainers to produce auto logous fibrin glue (AFG), which is used to create sticky bone.. The blood in the test tube is centrifuged at 2500 rpm using centrifuge for 3 minutes. With a syringe, AFG is obtained and combined with Autogenous bone graft mixed 1:1 with bovine bone and Allow for coagulation to take place for 5-10 minutes to develop sticky bone.

\section{CGF membrane preparation:}

20-60CC of patient's venous blood was taken from patients' vein in patient's forearm, and the blood is divided in to 4 silica coated tubes are centrifuged at $3000 \mathrm{rpm}$ using centrifuge for 15 minutes Following centrifugation, the silica covered tube reveals three distinct layers. The platelet deficient plasma is at the top, and the fibrin buffy coat layer, which is made up of a large and dense polymerized fibrin block containing concentrated growth factors, lies in the middle. The red blood cell layer is the lowest layer. (Kim, 2015)
Concentrated growth factors CGF is extracted from the test tube and placed in a metal storage box, which is then compressed with a metal cover to form CGF membranes that are used to cover the augmented site. Figure3-4

Finally, in both groups the flap was advanced and closed using a parallel mattress suture technique used in a double layer suturing method placed $5 \mathrm{~mm}$ from the incision line along with simple interrupted sutures using4-0 synthetic monofilament suture. Figure $5 *$

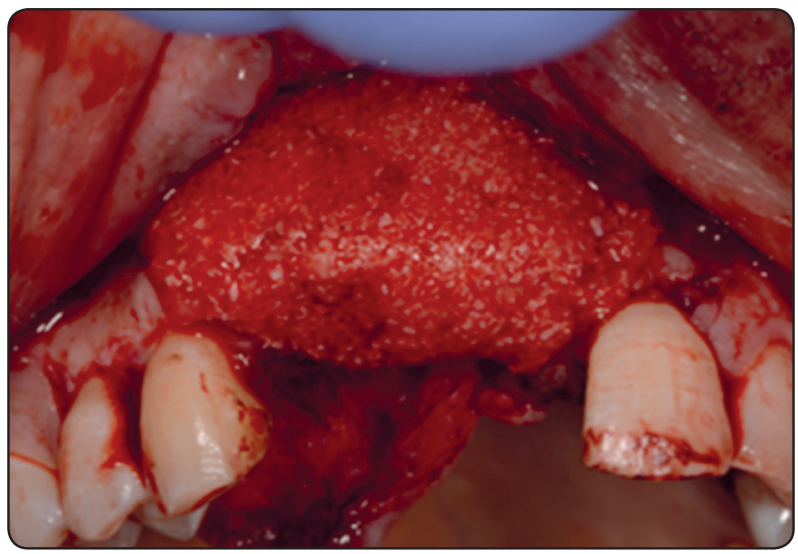

Fig. (3) sticky bone on bone defect

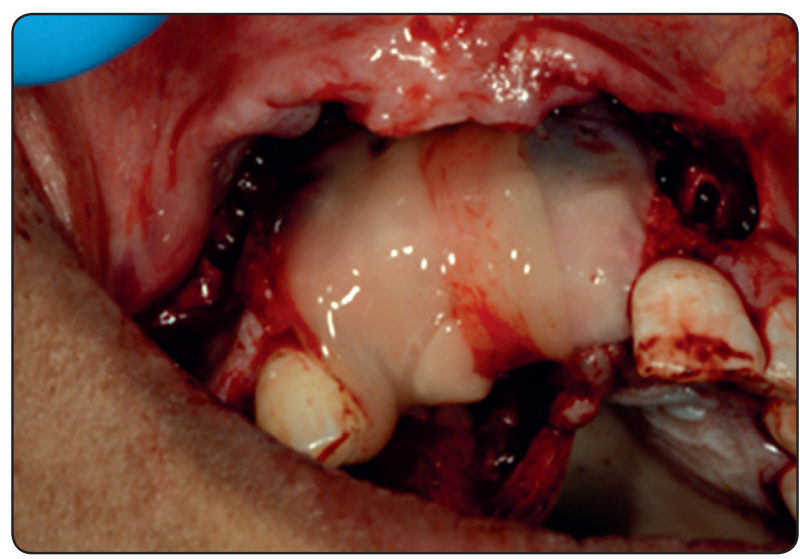

Fig. (4) CGF membrane placed on sticky bone

* Prolene, Assut, Switzerland. 


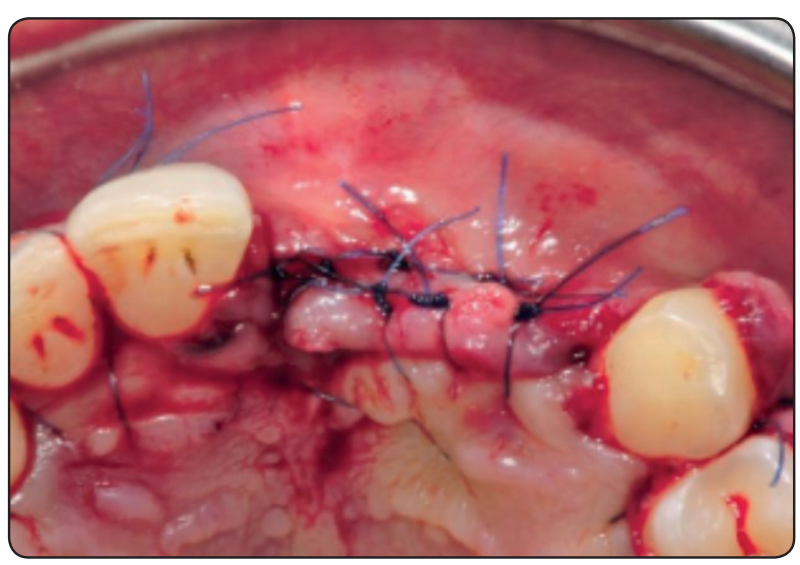

Fig. (5) Shows flap was sutured in a double layer double (Sticky Group)

\section{Post-operative care:}

- Post-operative medications will be prescribed as follow:

Amoxicillin/clavulanic acid tablets* $1 \mathrm{mg}$ every 12 hours for 7 days.

Non-steroidal anti-inflammatory analgesic (ibuprofen $400 \mathrm{mg}$ tablets) ${ }^{* *}$ every 8 hours for 4 days and then as needed

Antiseptic mouthwash (Chlorohexidine gluconate $0.2 \%$ ) $3^{* * *}$ times daily for 14 days beginning the day after the operation.

- Post-operative instruction will be explained to the patients as follows:

Starting the day following surgery, apply ice packs for 10 minutes every 30 minutes for 24 hours, and take strict oral hygiene measures such as brushing your teeth regularly and using antiseptic mouthwash.

\footnotetext{
* Augmentin 1 gram tab., Pfizer, United States of America

** Brufen 400MG 30tab. Abbott/Cairo, Egypt

*** Orovex mouthwash, Macro group, Egypt

**** Neo biotech Co., Ltd.

****** E-space Bldg., 36, Digital-ro 27 gil, Guro-gu, Seoul, 08381, Republic of Korea
}

Single interrupted sutures were released 10 to 14 days after operation, and mattress sutures two to three weeks afterwards.

\section{Follow up:}

Clinical evaluations were performed on all of the patients. at 1 week, 2 weeks, 1 month and 6 months post operatively.

Immediate post-operative CBCT scans were performed for all patients and at six-month periodically, to assess the amount of horizontal bone gain and calculate the percentage of graft resorption. a total of three scans were taken for each patient. All data were collected and tabulated for statistical analysis.

\section{2nd stage surgery}

After 6 months a flap was elevated to assess clinically the amount of bone formed Figure 6 and insertion of implants ${ }^{* * * *}$ with diameters ranging from $3.5-4.5 \mathrm{~mm}$.

Implant osteotomy preparation was under copious saline irrigation. Implant was inserted and placed proper prosthetic position.

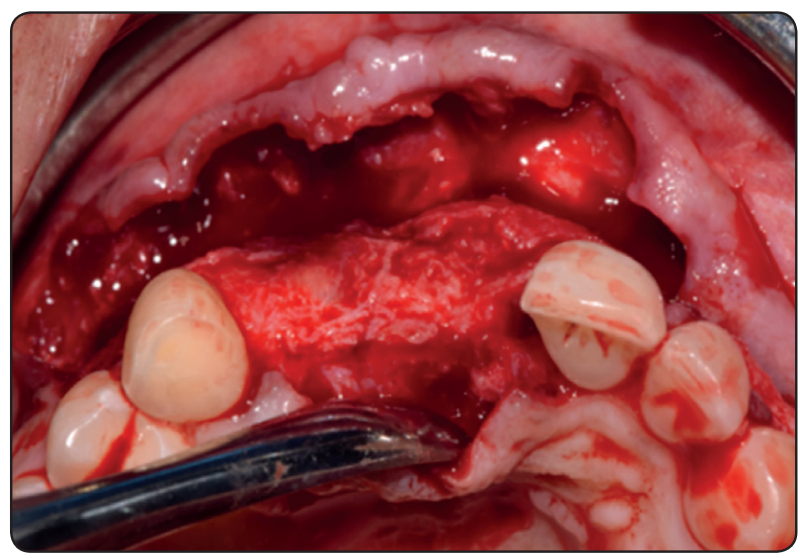

Fig. (6) 2nd stage surgery after 6 months 


\section{RESULTS}

This study evaluated the effect of two different guided tissue regeneration (GBR) techniques which are the lateral ridge augmentation using enriched bone graft matrix (sticky bone) covered by autologous concentrated growth factors (CGF) versus guided bone regeneration using native collagen membrane in horizontally deficient maxilla. The bone gain was determined using linear measurement via CBCT which were performed before surgical procedure, immediate and after six months. Implant stability was detected by using resonance frequency analysis via OSTELL which was performed immediately after surgical procedure.

The mean and standard deviation values were calculated for each group in each test. Data were explored for normality using Kolmogorov-Smirnov and Shapiro-Wilk tests. The data exploration revealed that normal parametric distribution (significant) was found in bone gain between sausage and sticky bone groups at level $1(2 \mathrm{~mm}$ crestal bone level). On the other hand, there was no parametric distribution (non-significant) in bone gain at level 2(5mm crestal bone level) and level 3 (10mm crestal bone level).

Independent sample t-test was used to compare between two groups in non-related samples. Paired sample t-test was used to compare between two groups in related samples. Two-way ANOVA was used to test the interaction between different variables.

The significance level was set at $\mathrm{P} \leq 0.05$. Statistical analysis was performed with IBM ${ }^{\circledR}$ SPSS ${ }^{\circledR}$ Statistics Version 20 for Windows.

The present study has included twenty-eight patients (12 female and 16 male) with mean age 39.5. After the completion of the follow up period (12ms), all outcomes related date was recorded and plotted for statistical analysis All patents were allocated to the assigned groups. One patient was allocated to CGF group, however, after drawing her blood sample it yields no CGF or CGF with poor quality she was transferred to the other group.

The healing time was 6 months. Radiological and clinical examination at the time of re-entry revealed integration of the grafts with the surrounding bone, without bone substitute loosening and/or particles in the flap except for two patients in CGF group were found that the graft material was resorbed and instead fibrous tissue was formed. For the other subjects, the gain in ridge dimension allowed a successful implant placement.

A statistically significant gain in alveolar ridge width was achieved at the crest $(2 \mathrm{~mm})$, midcrestal $(5 \mathrm{~mm})$ and apical $(10 \mathrm{~mm})$ levels.

For native collagen group, the median horizontal gain measured at 2,5 and $10 \mathrm{~mm}$ from the alveolar crest was 2.55 with range $(1.43,4.34) \mathrm{mm}, 2.61$ with range $(0.02,4.71) \mathrm{mm}$ and 1.15 with range $(-2.88$ ,4.89) $\mathrm{mm}$, respectively. While for CGF group, the median horizontal gain measured at 2,5 and $10 \mathrm{~mm}$ from the alveolar crest was 1.54 with range $(-0,97$, $2.93) \mathrm{mm}, 2.8$ with range $(-2.01,5.27) \mathrm{mm}, 2.97$ with range $(-1.32,5.4) \mathrm{mm}$, respectively. Table (1)

\section{Radiographic examination and evaluation:}

Cone beam computed tomography $(\mathrm{CB} / \mathrm{CT})$ * scans were obtained preoperatively, immediately after grafting, and 6 months after the grafting procedure. Volumetric and width measurements were performed to compare differences between the sticky bone group and sausage group Volumetric measurements of lateral ridge grafts were performed from all $\mathrm{CB} / \mathrm{CT}$ scans. The radiographs were made with the same machine and same exposure parameters. Image reconstruction was performed using special software*. All width measurements were made on tomographic slices perpendicular to the longitudinal axis of the alveolar crest, 2

* Planmeca promax- Finland, 15mA, 85 KV. 
$\mathrm{mm}, 5 \mathrm{~mm}$ and $10 \mathrm{~mm}$ from the top of the crest, at the anticipated implant sites. In the midline of the jaws, on the axial view, an anatomic reproducible landmark (e.g., the nasal spine) was defined and a straight line was drawn through it. The distance from the measuring point to this line, at the middle of the alveolar crest, was obtained using the software ruler. The same anatomic landmarks and distances were used for measurements on $\mathrm{CB} / \mathrm{CT}$ scans immediately after grafting and 6 months after the grafting procedure.

\section{Amount of Bone width gain:}

The results of Amount of Bone width gain in both groups presented in table 1

\section{Amount of Bone width gain}

At level 1(L1): the median bone gain of group A was 2.55 with range $(1.43,4.34) \mathrm{mm}$ compared to 1.54 with range $(-0.97,2.93) \mathrm{mm}$ in group $\mathrm{B}$. This was statistically significant $\mathrm{p}=0.017$; being lower in group B.

At level 1(L2): the median bone gain of group A was 2.61 with range $(0.02,4.71) \mathrm{mm}$ compared to 2.8 with range $(-2.01,5.27) \mathrm{mm}$ in group $\mathrm{B}$. This was statistically not significant $\mathrm{p}=0.71$.

At level 1(L3): the median bone gain of group A was 1.15 with range $(-2.88,4.89) \mathrm{mm}$ compared to 2.97 with range $(-1.32,5.4) \mathrm{mm}$ in group $\mathrm{B}$. This was statistically not significant $\mathrm{p}=0.168$.

TABLE (1) Mean, SD and independent $t$ test of bone width in the studied groups at different time intervals

\begin{tabular}{|l|c|c|c|c|c|}
\hline \multirow{2}{*}{ Time } & \multicolumn{2}{|c|}{$\begin{array}{c}\text { Group A (Sausage) } \\
\text { n=14 }\end{array}$} & \multicolumn{2}{c|}{$\begin{array}{c}\text { Group B (Sticky Bone) } \\
\text { P value }\end{array}$} \\
\cline { 2 - 5 } & Mean & SD & Mean & SD & \\
\hline Preoperative & 4.13 & 0.33 & 4.16 & 0.72 & 0.880 \\
\hline Immediate post-operative & 8.08 & 1.03 & 7.34 & 0.77 & 0.041 \\
\hline 6 months post-operative & 6.71 & 0.65 & 5.53 & 1.05 & 0.001 \\
\hline
\end{tabular}

SD: standard deviation, $P \leq 0.05$ is considered statistically significant, analysis done by Independent t test

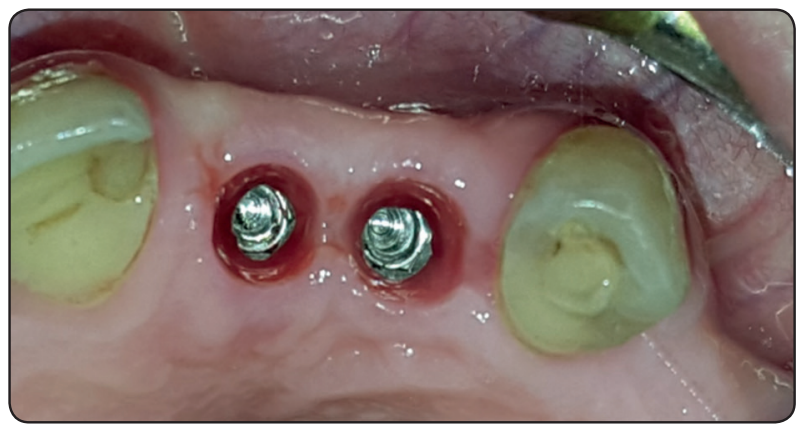

Fig. (7): Shows the healing of the soft tissue around the implant [sausage group]

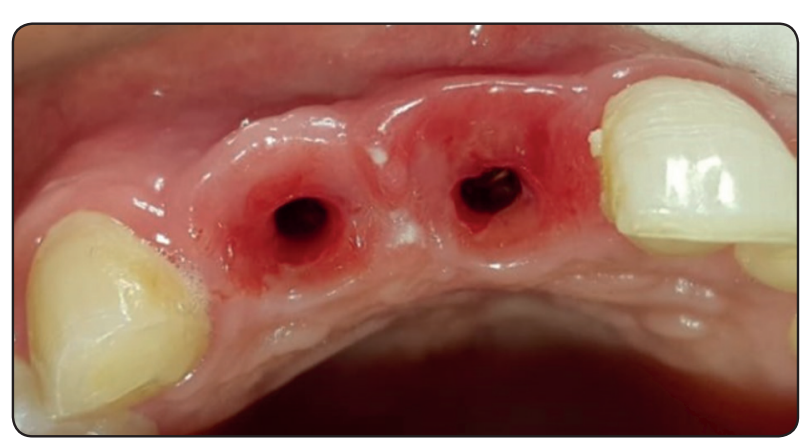

Fig (8): Shows the healing of the soft tissue around the implant [sausage group]

\footnotetext{
* Blue-sky plan software, Grayslake, United States of America
} 


\section{DISCUSSION}

GBR techniques for bone augmentation are widely documented and are characterized by great predictability and implant survival. The present study focused on radiographic changes of bone graft material volume evaluated by CBCT analysis after horizontal alveolar ridge augmentation by an Auto genous bone graft harvested from the retromolar area of the mandible and mixed with anorganic bovine bone matrix 1:1. The bone graft was covered either by a CGF membrane (test group) or by a resorbable collagen barrier membrane (control group). A concept of "Concentrated Growth Factors (CGF)" was introduced as third generation to Choukroun's PRF and gave the revolution of fabricating growth factors-enriched bone graft matrix (also known as "sticky bone") using autologous fibrin glue which has been demonstrated by Sohn 2010 Sticky Bone has the ability to be moldable and so well adapted over various shapes of bony defect. That prevent micro and macro movement of grafted bone, so the volume of bone augmentation is maintained during the healing period, therefore the need for block bone and titanium mesh is minimized. Fibrin network entraps platelets and leukocytes to release growth factors, so bone regeneration and soft tissue regeneration is enhanced. Another technique by Carlos et al2015 to utilize what called "steak bone" by injectable form of PRF called i-PRF. In this technique a short centrifuge for $2 \mathrm{~min}$ at $3300 \mathrm{rpm}$ gave an orange color fluid which can be injected or mixed with bone graft to give a well agglutinated "steak". Fibrin interconnection also minimizes soft tissue and epithelial cells ingrowth into sticky bone graft Sohn et al 2015 This was the reason why we used this protocol as a control group. We hypothesized that the CGF and PRF membranes could work as a scaffold or protective membrane both outwards to the periosteum and inwards to the augmented bone. The use of ABBM offers many advantages including adequate new bone formation, osteoconductive characteristics minimal resorption rate of $\mathrm{ABBM}$ compensating for the natural bone resorption caused by remodeling which preserves the graft's volume allowing good contact with the blood clot and interior passages that are interconnected allows cells and vessels to grow in agreement with Urban et al 2013. On the other hand, some authors found predictable bone width gain by using xenograft alone as reported by Simone et al 2018 mixed with leucocyte- and platelet-rich fibrin as it has low resorption rate and this may to be practically applied in CGF group not native collagen group as the collagen membranes had low resorption time to secure the underling graft materials. In present study the membrane used was bilayer non-cross-linked collagen membrane BioGide ${ }^{\circledR}$ which has good liquid uptake of ensures that growth factors and nutrients from the blood are taken up. The term "Concentrated Growth Factors (CGF)" was invented as third generation to Choukroun's PRF and gave the revolution of fabricating growth factors-enriched bone graft matrix (also known as "sticky bone") using Auto logous fibrin glue which has been developed by Sohn 2010 Sticky Bone has the ability to be moldable and so well adapted over a variety of bony defects. This prevents micro and macro movement of the grafted bone, retaining the volume of the bone augmentation during the healing process.

In present study, healing of soft tissue flaps was uneventful for all native collagen cases as well as CGF cases and no membrane exposures occurred at any of the surgical sites.

In present study, for collagen membrane group, the mean bone width was $4.13 \pm 0.33 \mathrm{~mm}$ giving an increase to $6.71 \pm 0.65 \mathrm{~mm}$. The results of the present study are in accordance with others reports in the literature indicate that the standard treatment for knife-edged ridges has changed in recent years. Nearly with the results were obtained by Hämmerle, 2008 ( $3.2 \mathrm{~mm}$ width increased to $6.9 \mathrm{~mm}) .92 \mathrm{In}$ addition, another study by Silvio Mario et al 2019 on Eighteen patients received 55 implants with horizontal bone width of $4 \mathrm{~mm}$ or less in mandible and maxilla were treated with 
resorbable collagen membranes and a 1:1 mixture of anorganic bovine bone and autogenous bone. After seven months, horizontal and volumetric bone dimensional changes measured on cone beam computer tomography (CBCT). The average horizontal bone gain of $5.03 \pm 2.15 \mathrm{~mm}$ which is higher than that in present study. The highest thickness gain was shown by Urban et al 2013, in a case series reported on 25 patients with $4 \mathrm{~mm}$ or less alveolar ridge width the clinical measurements revealed an average of $5.68 \mathrm{~mm} \pm 1.42 \mathrm{~mm}$ of lateral ridge augmentation. Candidates for the collagen membrane group presented with mean horizontal width of $4.13 \pm 0.33 \mathrm{~mm}$. While candidates for the sticky bone group had a mean horizontal width of $4.16 \pm 0.72 \mathrm{~mm}$. Regarding the base line width, there was no statistically significant difference between the two groups which indicates a random homogenous sample. After horizontal augmentation and a mean graft healing period of.6monthes. On the contrary to study reported by Simone et al 2018 evaluated the outcome of the leucocyte- and platelet-rich fibrin (L-PRF) Block for horizontal bone augmentation in the maxilla on ten patients, in the present study, the median horizontal bone gain of sticky bone group was 1.54 with range $(-0.97$, $2.93), 2.8$ with range $(-2.01,5.27), 2.97$ with range $(-1.32,5.4) \mathrm{mm}$ measured at 2,5 and $10 \mathrm{~mm}$ from the alveolar crest, respectively. This could be attributed to the difference between these study and our present study was that in our study the grafts were covered by only CGF membrane which has high resorption pattern than that of collagen membrane used in study mentioned above however, in none of the other studies, 93 compared or used sticky bone alone with CGF membrane.

Cagasan et al 2017 evaluate the effect of CGF on implant stability and osseointegration. Twelve patients were need implant in maxillary anterior region, implants cavities covered with CGF membrane before the implants are placed versus conventional implant placement. It accelerates the osseointegration of the implant. The mean ISQ values were found to be $79.40 \pm 2.604$ for the study group and $73.50 \pm 5.226$ for the control group at 1 st week, $78.60 \pm 3.136$ for the study group and $73.45 \pm 5.680$ for the control group at $4^{\text {th }}$ week . The rate of stabilization was positively significant. in present study, the mean ISQ values were found to be $65.00 \pm 5.32$ for collagen (control group) and $62.67 \pm 4.46$ for CGF (study group) at time of implant placement. After six months the mean ISQ values were found to be $73.43 \pm 4.39$ for collagen group and $72.83 \pm 6.05$ for the CGF. This result is in accordance with study mentioned above that the CGF has influence on implant osseointegration as well as the collagen membrane. The comparative study between collagen group and CGF group, showed that there is a significant difference in the median bone width gain between the two groups measured at $2 \mathrm{~mm}$ from the alveolar crest although, no significant difference in the median bone width gain at 5 and $10 \mathrm{~mm}$ measured from alveolar crest. Hence, the cause of the reduction in the graft may be attributed to resorption and remodeling of the bone particles, blood clot, and the rapid resorption of the CGF membrane compared to collagen membrane in combination with soft tissue pressure. Moreover, the results of this study showed that there were statistical differences raised between the mean bone width preoperative and postoperative in each group.

Owing to the fact that CGF is an autologous product, the availability of this bio-material in larger amounts is also a concern. One of the clinical limitations to deal with is the heterogeneity in the quality of platelets and blood components.At present, very little is understood about CGF generated from patients with coagulation disorders or patients on medications that affect blood clotting or platelet inhibitors. However, it was difficult to evaluate the CGF results of the current study because getting studies of the same outcomes and test group was unachievable. Therefore, from the available proofs gained from the results of this research it appears that the true effect of CGF on bone formation on 
horizontally deficient is questionable. Moreover, some patients have shown very poor CGF quality, and one patient whose blood failed to yield any CGF. This may shed a light on the potentiality of CGF and amount of growth factors represented within the sample drawn from each patient how many layers of CGF should to be placed and how to the degradation time to get the desired effect is a question that needs to be answered in order to achieve more standardized results.

\section{CONCLUSION}

Horizontal ridge augmentation using sticky bone covered by CGF membrane is unpredictable technique compared to guided bone regeneration using native collagen membrane. CGF can be considered a healing bio-material that can be utilized in regenerative surgical procedures to fasten healing, but its application as a barrier membrane in GBR is doubtful due to its poro echanical properties. GBR using a mix of autogenous bone graft with ABBM 50:50 covered by resorbable native collagen membrane is a predictable technique in horizontal bone augmentation in maxilla.

\section{REFERENCES}

1. Buser D. Thickness of the anterior maxillary facial bone wall-a retrospective radiographic study using cone beam computed tomography. Int J Periodontics Restorative Dent (2011) 31:125-131.

2. Cawood JI, Howell RA. A classification of the edentulous jaws. Int J Oral MaxillofacSurg. 1988; 17:232-236.

3. Cagasan Pirpir, C., Yilmaz, O., Candirli, C. "Evaluation of effectiveness of concentrated growth factor on osseointegration." International journal of implant dentistry 2017;3(1).

4. Hämmerle Advances in bone augmentation to enable dental implant placement: Consensus Report of the Sixth European Workshop on Periodontology. J Clin Periodontol. $2008 ; 35(8): 168-72$.

5. Juodzbalys, G., \& Kubilius, M. Clinical and radiological classification of the jawbone anatomy in endosseous dental implant treatment. Journal of oral \& maxillofacial research2013;4(2).
6. Jin Kim, DDS, MS W. Eric Park, DDS4• Charles C. Park DDS Utilization of Autologous Concentrated Growth Factors (CGF) Enriched Bone Graft Matrix (Sticky Bone) and CGF-Enriched Fibrin Membrane in Implant Dentistry 20155ce2249c7c5e2f337802a9e5_JIACD sticky bone December 2015.

7. Lindhe J, Mauricio G. Arau'jo Flavia Sukekava Jan L. Wennstro“m. Tissue modeling following implant placement in fresh extraction sockets. Clin Oral Implants Res. 2006;17(6):615-24.

8. Manuel Vallecillo-Capilla Measurement of dental implant stability by resonance frequency analysis: A review of the literature. Journal section: Oral Surgery. $2009 ; 14$ (10):e538-46.

9. Silvio M Mario, Jovanovic Sascha A, IstvanU, Luigi C, Milena P, MarcoT, Horizontal Ridge Augmentation using GBR with a Native Collagen Membrane and 1:1 Ratio of Particulated Xenograft and Autologous Bone: A 1-Year Prospective Clinical Study Clinical Implant Dentistry and Related Research 2017;19, 1,

10. Col. Bras. Cir. 42 (6) Nov-Dec 2015 Obtention of injectable platelets rich-fibrin (i-PRF) and its polymerization with bone graft: technical note.

11. Schropp, L., Kostopoulos, L. \& Wenzel, A. Bone healing following immediate versus delayed placement of titanium implants into extraction sockets: a prospective clinical study. International Journal of Oral Maxillofacial Implants (2003a,b) 18: 189-199.

12. Simone Cortellini Ana Castro Andy Temmerman Rutger Dhondt Jeroen Van Dessel Reinhilde Jacobs Marc Quirynen L-PRF block for horizontal bone augmentation, clinical results and histology- a prospective case series. Clin Oral Impl Res. 2018;29(17).

13. Sohn DS. Lecture titled with sinus and ridge augmentation with CGF and AFG, Symposium on CGF and AFG, 2010.

14. Sohn DS. Clinical Applications Incorporating the use of Concentrated Growth Factors (CGF/PRF) to Enhance Healing, Maturation, and Predictability and to Simplify Complex Procedures Journal of Implant and Advanced Clinical Dentistry 2015; 11-29.

15. Urban, Istvan A. "Horizontal ridge augmentation with a collagen membrane and a combination of particulated autogenous bone and anorganic bovine bone-derived mineral: a prospective case series in 25 patients.” International Journal of Periodontics\& Restorative Dentistry (2013).33.3. 